\title{
Application of an Opto-Electronic Oscillator in 5G Mobile and Wireless Networks with a Low Frequency Drift, a High Side-Modes-Suppression Ratio and without a Power Penalty due to Chromatic Dispersion
}

\author{
Mehmet Alp Ilgaz, Boštjan Batagelj \\ University of Ljubljana, Faculty of Electrical Engineering, \\ Tržaška cesta 25, 1000 Ljubljana, Slovenia \\ e-mail: mehmet.ilgaz@fe.uni-lj.si
}

\begin{abstract}
This paper describes and discusses the practical application of an opto-electronic oscillator for the next generation of $5 \mathrm{G}$ mobile and wireless networks based on millimeterwave technology. The application of a single-loop, opto-electronic oscillator is designed for 39 $\mathrm{GHz}$ and will be implemented in the central-station of an upcoming $5 \mathrm{G}$ mobile and wireless network. The aim of this configuration is to decrease the complexity of the base-stations for a next-generation system and provide an economic advantage for upcoming $5 \mathrm{G}$ networks. The system is designed to avoid the power penalty due to the chromatic dispersion, is temperature stabilized to have a long-term stability and has a low side-modes effect to ensure a low phase noise.
\end{abstract}

Keywords-5G wireless and mobile networks; low phase noise; single-loop opto-electronic oscillator; frequency drift; side-modes suppression ratio; power penalty

\section{INTRODUCTION}

The opto-electronic oscillator (OEO) is one of the well- known technologies that can generate high-frequency signals in the micro-wave and millimeter-wave ranges. It was designed in the mid-1990s and has seen many modifications and improvements in recent decades [1]. One of the main advantages of the OEO is its relatively low phase noise, together with it independence from the operating frequency [2]. An OEO consists of optical and electrical components (Fig. 1) and there is a possibility to obtain the optical and electrical outputs simultaneously [3].

The next generation of $5 \mathrm{G}$ mobile and wireless networks will require a high spectrum efficiency with a low data latency. For this reason, a lot of effort has been put into $5 \mathrm{G}$ radio access network efficiency [4]. Due to the demand for a high data bit rate, the millimeter-wave frequency band will probably be used and the number of base-stations will increase.

Currently, the spectrum efficiency is low in the range of millimeter waves, because the phase noise of current oscillators in this range is poor and consequently the sub-carrier spacing needs to be high [5]. Therefore, low-phase-noise oscillators are becoming more and more important for $5 \mathrm{G}$ networks in the millimeter-wave frequency band.

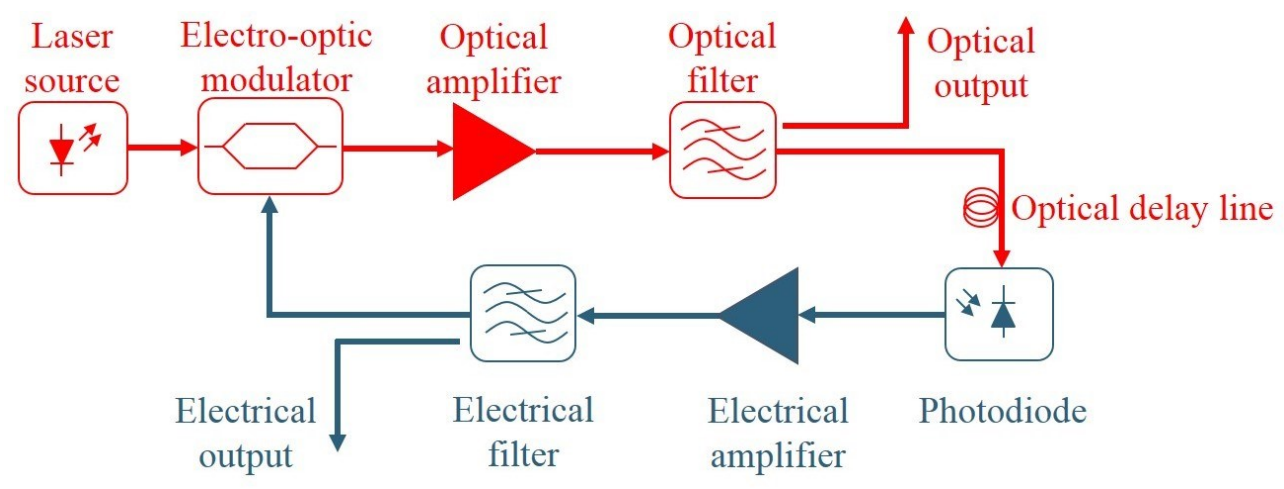

Fig. 1. Schematic of a single-loop OEO. In the OEO, the optical filter, optical amplifier and/or electrical amplifier and filter can be placed in the loop.

As was mentioned before, an OEO can be used to produce a low-phase-noise, millimeterwave signal. Since an OEO is too costly and sensitive to environmental perturbations, it 
cannot be placed in every base-station, as is the case with electrical oscillators in today's 4G networks. One idea [6] to solve this dilemma and use OEOs in 5G mobile and wireless networks is to place it at the core of the network. The idea, shown in Fig. 2, can be explained in terms of the OEO being placed in the central-station of the $5 \mathrm{G}$ mobile network instead of placing each local oscillator in the base-stations.

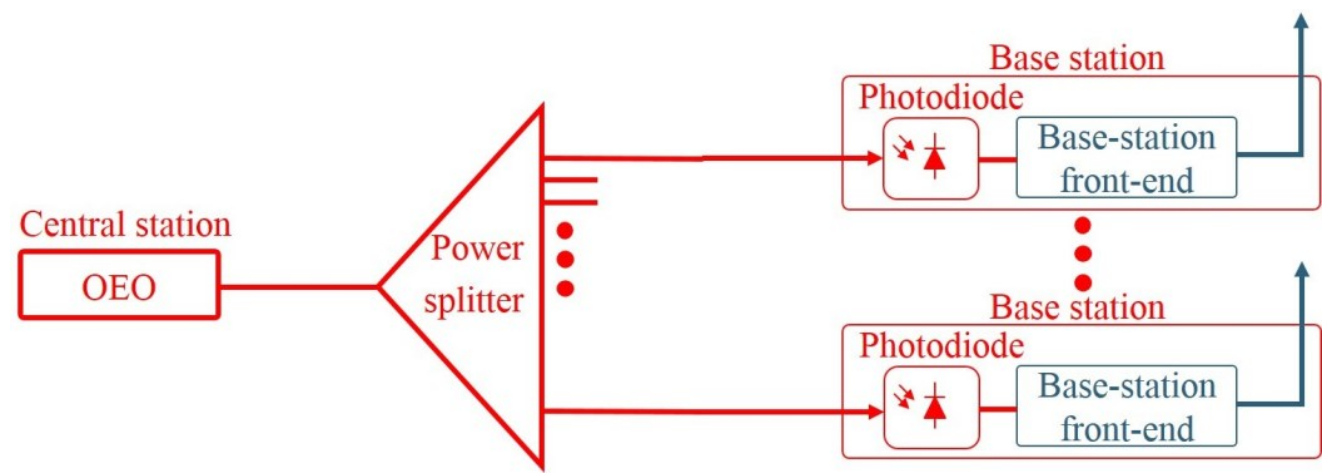

Fig. 2. The idea of the OEO's signal distribution from the central-station to the base-station front end [6].

With the help of the centralized oscillator idea, there is only one signal oscillator used for the whole network. One of the advantages of this application is that all the base-stations receive the same signal and they can use it for the synchronization between different nodes. We believe that with such high phase stability the latency of a $5 \mathrm{G}$ network can be reduced.

Since the OEO is placed in the temperature-controlled environment of the central-station, it will provide a stabilized high-frequency signal to the base-stations. In addition, with this idea it will help us to simplify the base-stations of the $5 \mathrm{G}$ network. This means that we do not even need a local oscillator (LO) for each base-station for the purposes of signal up and down conversions. We can remove the LO from each base- station and place one OEO in the centralstation and provide its stabilized signal to each base-station.

The OEO has a high $Q$ factor that is proportional to the length of the delay line. This means that a longer optical fiber gives a higher $Q$ factor and a low phase noise. Fig. 3 shows the phase-noise behavior of the same OEO loop with a different optical fiber length. The 15-km fiber length of the OEO has a lower phase noise due to having a high $Q$ factor compared to the 1-km optical fiber OEO [7].

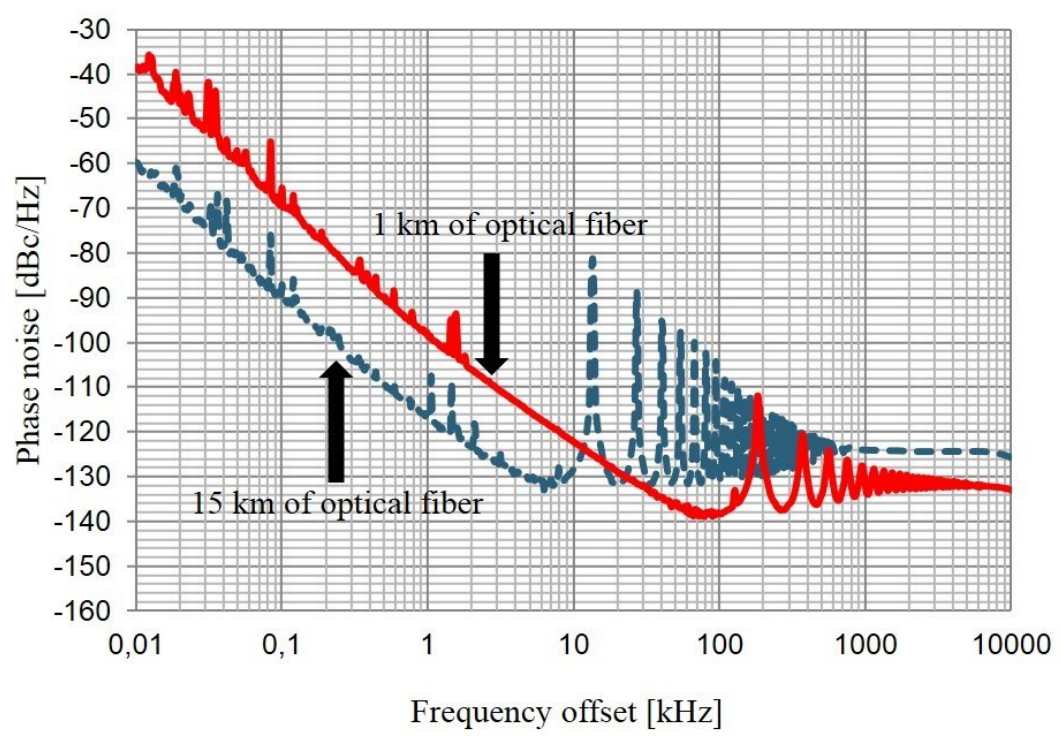

Fig. 3. Comparison of the phase noise for OEOs with $1 \mathrm{~km}$ and $15 \mathrm{~km}$ of optical fiber [7]. 
Even though there are some advantages of having an OEO in a $5 \mathrm{G}$ mobile and wireless network, there are some challenges to affect the performance of the OEO in the millimeterwave signal range. One of the well-known problems is the power penalty due to the optical fiber's chromatic dispersion of the OEO. This problem can cause a phase shift of the highfrequency signal from the central-station to the base- station and in some cases it causes the total destruction of the signal transmission. Another problem is called the frequency drift [8]. The reason for this frequency drift is the unexpected temperature changes with respect to the oscillator's loop. These changes in the temperature will affect the frequency behavior of the oscillator. This means that the oscillator cannot oscillate at the main frequency. In addition, this frequency drift can cause mode hopping [8].

Another problem with the OEO is known as spurious modes [8]. The reason for the side modes is the non-ideal behavior of the filter of the OEO. This means that the electrical/optical filter of the oscillator loop does not ideally destroy all the other modes of the oscillator. They are attenuated, but can still be seen.

In the next section we discuss how to overcome the practical problems of chromatic dispersion, frequency drift and side modes for the implementation of an OEO in a 5G mobile and wireless network. The system's design and performance evaluation are presented using a prelaminar experimental proof of the concepts and simulations.

\section{IMPLEMENTATION OF AN OEO IN A 5G NETWORK}

Designing an efficient single-loop OEO for a 5G mobile and wireless network in the millimeter-wave frequency band is not an easy task. Each component of the OEO loop affects the performance of the system. The specific problems/challenges of the OEO are depicted in Fig. 4. This can be separated into four sections. These are the frequency drift, the multi-mode operation, the chromatic dispersion effect and the Rayleigh scattering. The OEO should be designed accordingly so that it can work efficiently in the millimeter-wave range. Fig. 4 shows these problems in a single-loop OEO. The phase noise of the OEO generally depends on the laser's relative intensity noise (RIN), the shot noise, the thermal noise and the amplifier noise. In addition, Rayleigh scattering and the modulation technique can contribute to the phasenoise floor of the OEO.

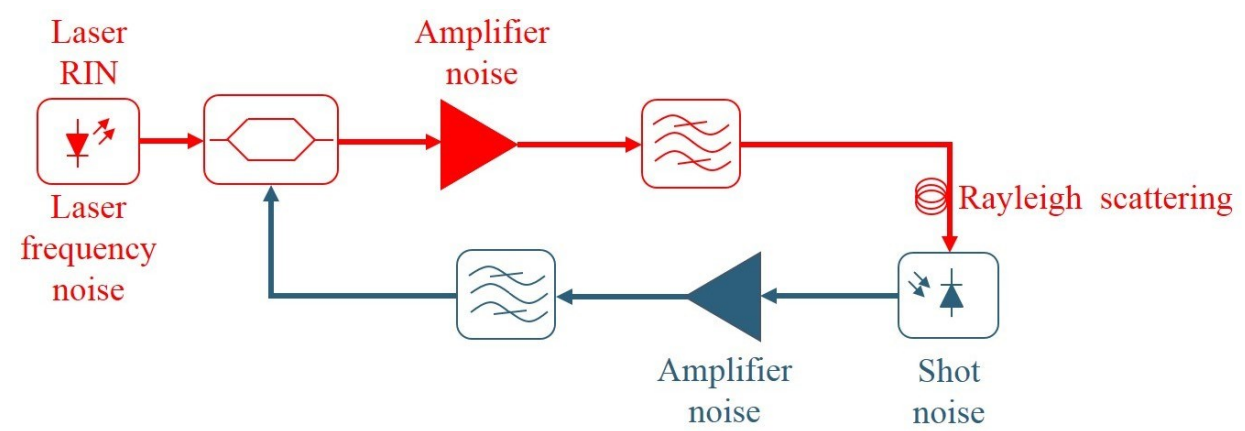

Fig. 4. Main contributions of phase noise in the single-loop OEO as specified from the components [9-10].

One of the critical challenges when it comes to implementing a single-loop OEO in a 5G mobile and wireless network is the spurious modes. These modes occur due to the non-ideal behavior of the electrical filter. To eliminate these unwanted modes, some methods have been invented. One of the popular techniques is to insert another optical delay line, called a dual-loop OEO [11] or multi-loop OEO [12-13]. In addition, there is another method called an injection-locked OEO [14] that is also useful for suppressing the side modes of the oscillation. Another promising solution is to use an ultra- high finesse etalon in the OEO's loop [15]. This optical filter has a very high Q-factor to destroy the side modes.

However, these methods [11-15] have some economic and technical disadvantages for $5 \mathrm{G}$ 
mobile and wireless networks. The dual- or multi-loop configuration of the OEO in $5 G$ networks has a technical disadvantage in that the size of the OEO is increased with an additional optical delay line (fiber spool and photodiode) and an injection-locked OEO requires very precise tuning. A finesse etalon [15] is not easily found on the market and it is very expensive, which means that it increases the cost of the OEO in $5 \mathrm{G}$ mobile and wireless networks. A useful method that could be implemented in an OEO is an OEO with a quality multiplier [16]. The idea of a quality multiplier in millimeter-wave range applications is shown in Fig. 5.

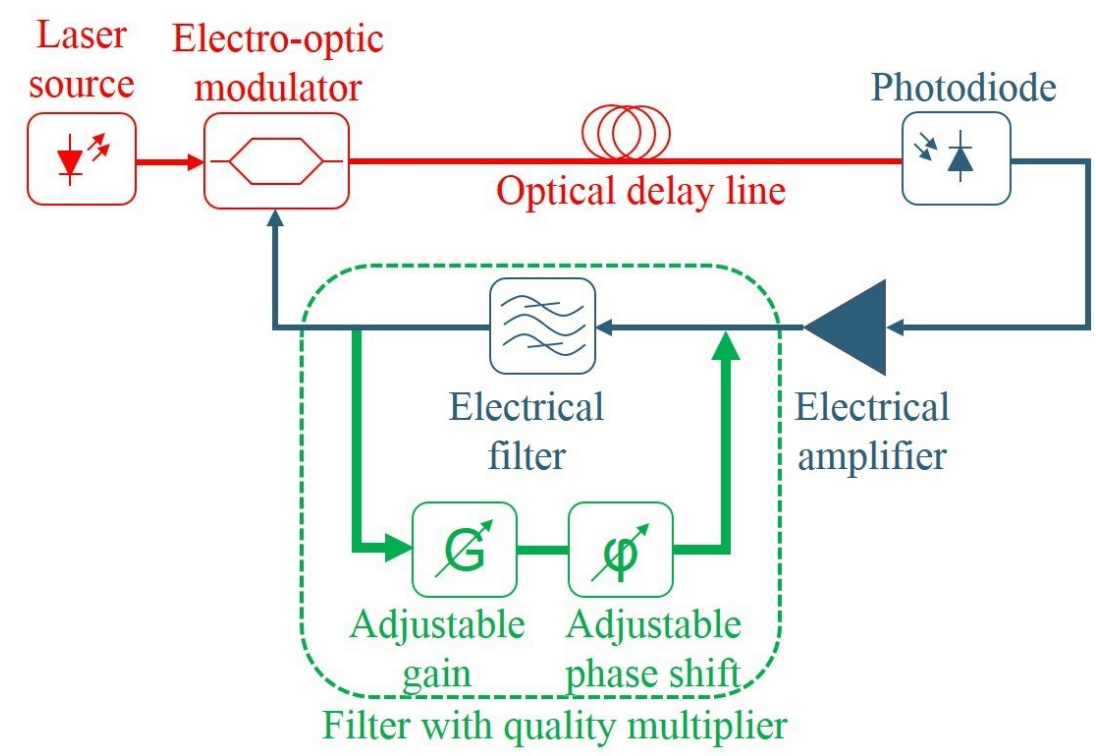

Fig. 5. Schematic of a single-loop OEO with a quality multiplier that will be built up in the millimeter-wave range [16].

With this technique [16], an electrical circuit is connected to the bandpass filter of the OEO to decrease the bandwidth of the OEO and to increase the $Q$ factor of the loop. The narrower the bandwidth, the higher the $Q$ factor that is obtained. Therefore, the side modes of the oscillation are more suppressed. In our prelaminar proof of concept with a $3-\mathrm{GHz}$ setup, a $20-\mathrm{dB}$ improvement of the SMSR was obtained.

Another practical challenge with an OEO implementation in $5 \mathrm{G}$ mobile and wireless networks is called the frequency drift. This problem is very critical and affects the oscillator's performance. One possible solution is to implement a feedback control loop in the OEO [17]. With this idea, a feedback control loop is inserted into the single-loop OEO. The feedback control loop consists of a frequency discriminator and a proportional-integral controller. A feedback control loop keeps the frequency constant, because it makes the group delay of the oscillator's loop constant. The group delay of the OEO's loop depends on factors such as the refractive index and amplifier's phase shift. If the group delay changes, the control loop will compensate for this change with a changed refractive index. As a result, the group delay, and therefore the frequency, will remain constant. With this method a frequency drift of $0.05 \mathrm{ppm} / \mathrm{K}$ is obtained in our prelaminar experimental proof of concept. In comparison, the frequency drift of a free-running OEO is about $8 \mathrm{ppm} / \mathrm{K}$. Another possibility is to insert an additional fiber delay monitoring mechanism [18]. With our idea for the implementation of the OEO in the centralstation, the temperature stabilization of the optical fiber and the electrical filter of the OEO is easier than in the base-station. Besides the advantage of the long-term stability enhancement of the OEO, this stabilization technique is not adding any additional phase noise to the oscillator signal.

Moreover, the power penalty due to the chromatic dispersion is another critical practical challenge to affect the signal's transmission over an analog optical link. Fig. 6 shows the experimental setup to transfer the signal from $10 \mathrm{MHz}$ to $40 \mathrm{GHz}$ via an analog optical link over a 5-km, 10-km and 15- km optical fiber. As is clear from Fig. 7, there are three deep points occurring for the 15-km length of the optical fiber. 


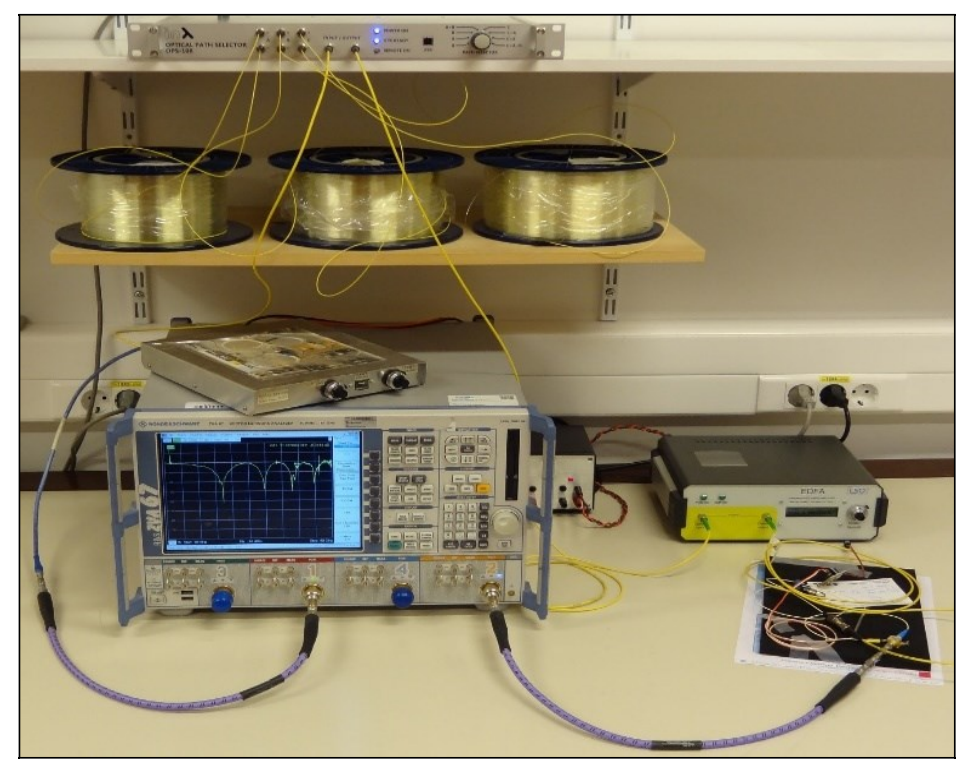

Fig. 6. Experimental setup for the measurement of dispersion induced power penalty.

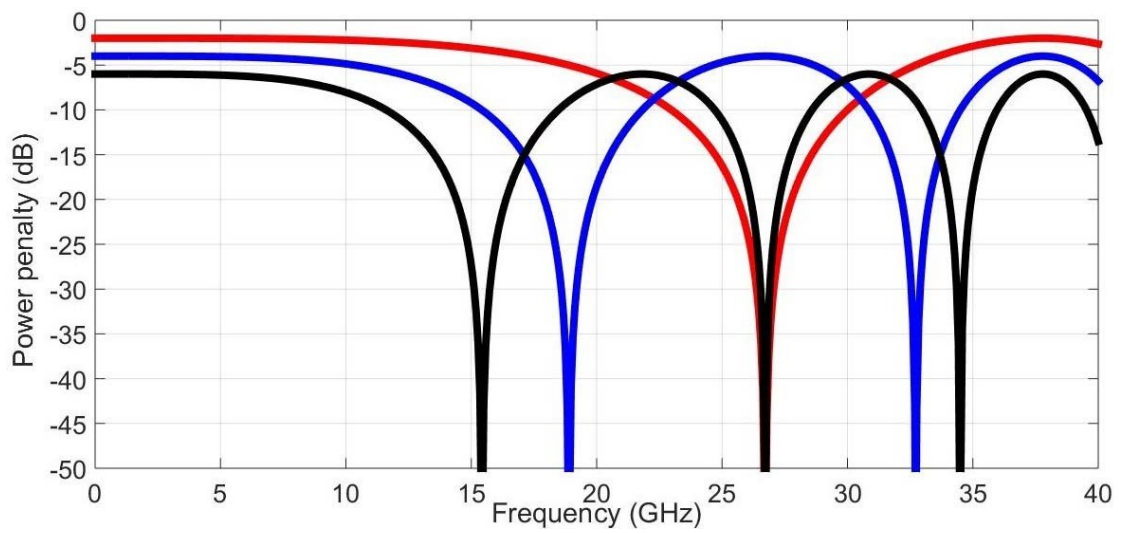

Fig. 7. Power-penalty simulation of the signal from $10 \mathrm{MHz}$ to $40 \mathrm{GHz}$ with an optical fiber length of $5 \mathrm{~km}$ (red), $10 \mathrm{~km}$ (blue) and $15 \mathrm{~km}$ (black).

The results in Fig. 7 highlight one of the critical parameters in the design of a single-loop OEO for $5 \mathrm{G}$ mobile and wireless networks. In other words, the desired operating frequency and the optical length from the central-station to the base-stations needs to be carefully designed in order to avoid a power penalty. This power penalty occurred due to the chromatic dispersion of the optical fiber. In addition, chromatic dispersion is dominant above the $20 \mathrm{GHz}$ [19].

In order to beat the chromatic dispersion effect on the OEO, there have been some methods reported in the literature. One of the best-known techniques is to use a dual-drive MachZehnder modulator to produce a single side band (SSB) signal. With this technique, the authors did not see the chromatic dispersion effect up to $79.6 \mathrm{~km}$ [20], which is more than enough for radio access network fronthaul. This is a good technique to avoid chromatic dispersion, but unfortunately it increases the system's complexity and therefore the price.

In addition, there are special optical fibers used instead of the single-mode fiber G.6552D to avoid the chromatic dispersion. They are called dispersion-shifted fiber (DSF) [21] or dispersion compensated fiber (DCF) [22]. These solutions are also not compatible with 5G mobile and wireless networks because the passive optical networks (PONs) between the central- and base-stations are already places with a standard single-mode fiber. To replace a PON with a DCF or a DSF is an expensive solution that also requires extra time and for this reason we cannot afford it in practice. One of the most useful solutions is to use a 1310-nm 
wavelength laser. In the 1310-nm wavelength, the dispersion coefficient is very close to zero and its effect is almost negligible.

The performance evaluation, which is a simulation, is shown in Fig. 8. It is clear from the simulation that there is no power penalty due to the chromatic dispersion. There is some loss when the optical length is increased. This is normal because the optical loss is directly related to the length of the optical fiber.

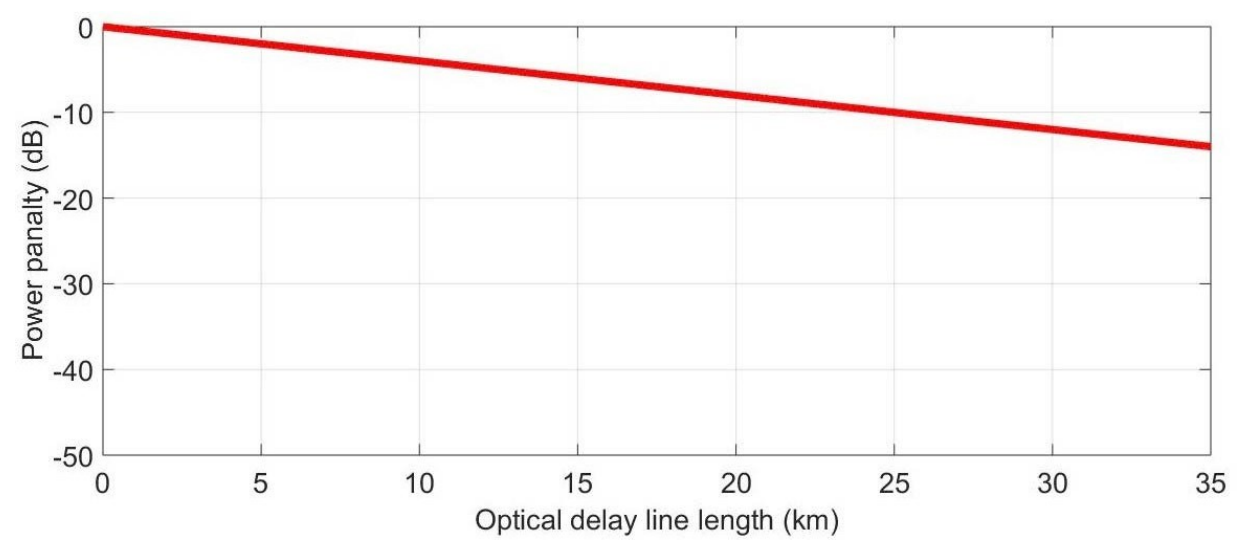

Fig. 8. Simulated results of the power penalty depend on the optical length between 5 and $35 \mathrm{~km}$ (increment of $5 \mathrm{~km}$ ) with $1310 \mathrm{~nm}$ for the $39-\mathrm{GHz}$ frequency.

One of the drawbacks of using a 1310-nm laser is that the optical loss is more than with the $1550-\mathrm{nm}$ laser wavelength. The optical loss is about $0.35 \mathrm{~dB} / \mathrm{km}$ with the $1310-\mathrm{nm}$ wavelength, while the loss is $0.2 \mathrm{~dB} / \mathrm{km}$ with the $1550-\mathrm{nm}$ wavelength.

As a final evaluation, to have a stable opto-electronic oscillator for $5 \mathrm{G}$ mobile and wireless networks, a temperature- stabilized optical fiber and RF electrical filter combined with the quality-multiplier method using a 1310-nm wavelength can be used. This combined system provides a low-phase-noise signal without any power penalty to the base-station via a passive optical network. Moreover, there could be a semiconductor optical amplifier placed on the PON to compensate for the optical losses. The main advantage of this complex system compared to the other methods mentioned above (such as dual-loop OEO or others) is that it uses cheaper and less-complex structures.

\section{CONCLUSION}

In this paper a useful application is described and explained for use in upcoming $5 \mathrm{G}$ mobile and wireless networks operating in the millimeter-wave range. These networks require high bandwidth, low latency, low phase noise and high speed. An OEO might be a good oscillator that could be implemented in the central-station to decrease the complexity of the basestations.

In addition, the OEO has a low phase noise compared to other technologies such as the airdielectric resonator, quartz- crystal oscillators, etc. One of the main advantages of the OEO mentioned in the introduction to this paper is that the phase noise is not directly related to the operating frequency. In other words, the phase-noise characteristic of the OEO stays the same if we move the frequency from the micro-wave region to the millimeter-wave region where the $5 \mathrm{G}$ mobile and wireless network needs to meet the high standards of the specifications.

The possible practical challenges are described according to the literature and past experiences with opto-electronic technology for the implementation of an OEO in a $5 \mathrm{G}$ mobile and wireless network based on millimeter-wave technology. According to our idea, the OEO application in the central- station, with the decreasing complexity of the base-stations in the network, is an effective solution for bringing the synchronization between the nodes. On other hand, there are some issues described to affect the performance of the OEO. These topics must also be comprehensively taken into account when the OEO is used in a 5G network. 
The effect of the problems/challenges mentioned should be minimized as much as possible to increase the performance of the OEO in $5 \mathrm{G}$ mobile and wireless networks.

\section{ACKNOWLEDGMENT}

The authors would like to express their gratitude to the company InLambda BDT d.o.o. for the research equipment and devices used for the preliminary proof of concept. The work presented in this article was carried out within the FiWiN5G Innovative Training Network, which has received funding from the European Union's Horizon 2020 Research and Innovation Programme 2014-2018 under the Marie Skłodowska-Curie Action grant agreement No.642355. The authors also acknowledge the financial support of the Slovenian Research Agency (research core funding No. P2-0246).

\section{REFERENCES}

[1] T. Hao, J. Tang, D. Domenech, W. Li, N. H. Zhu, J. Capmany, M. Li, "Towards monolithic integration of OEOs: From systems to chips," J.

Lightwave Technol., April 2018, DOI:10.1109/JLT.2018.2825246

[2] B. Batagelj, L. Bogataj, and M. Vidmar, "Key properties and design issues for an optoelectronic oscillator," in Proc. ICTON 2015, pp.1-4, Budapest, Hungary, July 2015, paper Mo.C5.4.

[3] A. Madjar and T. Berceli, "Microwave generation by optical techniques - a review," Proc. EUMC, Manchester, United Kingdom, 2006, pp. 1099-1102.

[4] C. L. I, H. Li, J. Korhonen, J. Huang and L. Han, "RAN Revolution With NGFI (xhaul) for 5G," J. Lightwave Technol., vol. 36, no. 2, pp. 541-550, Jan. 2018.

[5] A. A. Zaidi, R. Baldemair, H. Tullberg, H. Bjorkegren, L. Sundstrom, J. Medbo, C. Kilinc, I. D. Silvaet, "Waveform and Numerology to Support 5G Services and Requirements," IEEE Communications Magazine, vol. 54, no. 11, pp. 90-98, November 2016.

[6] M. A. Ilgaz, and B. Batagelj, "Proposal for the distribution of a low- phase-noise oscillator signal in the forthcoming fifth-generation mobile network by radio-over-fibre technology," ELMAR 2016, pp.13-16, Zadar, Croatia, Sep. 2016.

[7] M. A. Ilgaz, and B. Batagelj, "Preliminary idea for a converged fixed and mobile network infrastructure with 5G using Radio-over-Fiber technology and an Opto-Electronic Oscillator in the millimeter-wave range," Proc. ICTON 2016, pp.1-4, Trento, Italy, July 2016, paper Tu.P.5.

[8] L. Bogataj, M. Vidmar, and B. Batagelj, "A feedback control loop for frequency stabilization in an opto-electronic oscillator," J. Lightwave Technol., vol. 32, no. 20, pp. 3690-3694, Oct. 2014.

[9] M. A. Ilgaz, and B. Batagelj, "Opto-Electronic Oscillator in the mm-W range for $5 \mathrm{G}$ Wireless and Mobile Networks: Design Challenges and Possible Solutions," ONDM 2017, pp.1-5, Budapest, Hungary, May 2017.

[10] A. Docherty, C. R. Menyuk, J. P. Cahill, O. Okusaga, and W. Zhou, "Rayleigh-ScatteringInduced RIN and Amplitude-to-Phase Conversion as a Source of Length-Dependent Phase Noise in OEOs," IEEE Photonics Journal, vol.5, no.2, Apr. 2013.

[11] J.-H. Cho, H. Kim, and H.-K. Sung, "Reduction of Spurious Tones and Phase Noise in Dual-Loop OEO by Loop-Gain Control," IEEE Photonics Technology Letters, Vol. 27, no.13, pp. 1391-1393, July 2015.

[12] D. Eliyahu and L. Maleki, "Low phase noise and spurious level in multi- loop optoelectronic oscillators," Proc. IEEE Int. Freq. Control Symp./PDA Exhib., Florida, USA, , pp. 405-410, 2003.

[13] X.S. Yao and L. Maleki, "Multiloop optoelectronic oscillator," IEEE J. Quantum Electr., vol. 36 , no. 1, pp. 79-84, Jan. 2000.

[14] W. Zhou and G. Blasche, "Injection-locked dual opto-electronic oscillator with ultra-low phase noise and ultra-low spurious level," IEEE Trans. Microw. Theory Techn., vol. 53, no. 3, pp. 929-933, Mar. 2005.

[15] I. Ozdur, D. Mandridis, N. Hoghooghi, P. J. Delfyett, "Low noise optically tunable opto- 
electronic oscillator with Fabry-Perot etalon," Journal of Lightwave Technology, vol. 28, pp. 3100-3106, Nov. 2010.

[16] L. Bogataj, M. Vidmar, and B. Batagelj, "Opto-electronic oscillator with quality multiplier," IEEE Trans. Microw. Theory Techn., vol. 64, no. 2, pp. 663-668, Feb. 2016.

[17] L. Bogataj, M. Vidmar, and B. Batagelj, "Improving the Side-mode Suppression Ratio and Reducing the Frequency Drift in an Opto- Electronic Oscillator With a Feedback Control Loop and Additional Phase Modulation," J. Lightwave Technol., vol. 34, no. 3, pp. 885-890, Feb., 12016.

[18] W.-H. Tseng and K.-M. Feng, "Enhancing long-term stability of the optoelectronic oscillator with a probe-injected fiber delay monitoring mechanism," Optics express, vol. 20, no. 2, pp. 1597-1607, Jan. 2012.

[19] U. Gliese, S. Ngrskov, and T. N. Nielsen, "Chromatic Dispersion in Fiber-Optic Microwave and Millimeter-Wave Links," IEEE Trans. Microw. Theory Techn. vol.44, no.10, pp.1716-1724, Oct.1996.

[20] G. H. Smith, D. Novak, and Z. Ahmed, "Overcoming chromatic- dispersion effects in fiber-wireless systems incorporating external modulators," IEEE Trans. Microw. Theory Techn., vol. 45, no. 8, pp. 1410-1415, August.1997.

[21] E. Salik, N. Yu, M. Tu and L. Maleki, "EDFA-based coupled opto- electronic oscillator and its phase noise," IEEE OFC 2004, vol.2, pp.1-3, Los Angeles, USA, Feb. 2004.

[22] C. Williams, D. Mandridis and P. J. Delfyett, "Noise Characterization of an InjectionLocked COEO With Long-Term Stabilization," J. Lightwave Technol., vol. 29, no. 19, pp. 2906-2911, Oct. 2011. 